\title{
Enlarging the Control Space of the Pyramid Wavefront Sensor: Numerical Simulations and Bench Validation
}

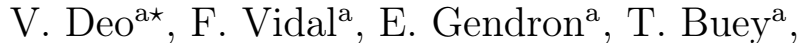 \\ D. Gratadour ${ }^{\mathrm{a}}$, Z. Hubert ${ }^{\mathrm{a}}, \mathrm{M}$. Cohen ${ }^{\mathrm{b}}$, and G. Rousset ${ }^{\mathrm{a}}$ \\ ${ }^{\text {a}}$ LESIA, Observatoire de Paris - CNRS - UPMC - Univ. Paris 7 Diderot \\ 5 place Jules Janssen, 92190 Meudon, France \\ ${ }^{\mathrm{b}}$ GEPI, Observatoire de Paris - PSL Research University - CNRS \\ 61 avenue de l'Observatoire, 75014 Paris, France
}

\begin{abstract}
We investigate the currently experienced limitations regarding the use of the Pyramid Wavefront Sensor (PWFS) in the coming generation of very high order adaptive optics (AO) systems for ELTs.

As a focal plane wavefront sensor, the PWFS concept paves way for a telescope diffraction limited AO regime, hence potential 80 times or more sensitivity improvement over a comparable Shack-Hartmann (SH) on large telescopes. Although some theoretical similarities exist with quad-cell SHs, the focal/pupil planes inversion brings up significantly different modelizations. Diffractive effects between PWFS pupil images (quadrants), nonlinear behavior -limited dynamic range, optical gain fluctuations- are PWFS features that require AO systems and controllers be specifically engineered. Demonstration of physical validity and robustness of PWFS models on sky-ready, ELT-scaled AO systems remains an open topic to date.

Obtaining a high quality pyramidal prism and a model-consistent WFS assembly is noticeably a critical factor. We demonstrate that the traditional gradient sensing controller is extremely sensitive to prism shape defects and common assembly misalignments: we generically analyze the effect of sensor-plane quadrants individual translations, which are induced by a variety of mechanical defects.

Quadrant misregistrations displace wavefront information to terms not included in the conventional slopes maps, and high spatial frequencies become invisible to the WFS. Expanded Space Control (ESC) for quad-cell signal incorporates additional WFS signals in addition to the X- and Y-axis differences, and compensates misalignmentinduced information loss, therefore dramatically relaxing machining and alignment constraints for PWFS engineering. We here present validating results of ESC performance and robustness for quadrant misregistration cases, both in end-to-end simulations with COMPASS and on the PYRCADO PWFS demonstrator bench at LESIA.
\end{abstract}

Keywords: Pyramid Wavefront Sensor, Extremely Large Telescope, Modal Control, Wavefront Reconstruction.

\section{INTRODUCTION}

The present paper is a progress report of ongoing research about Pyramid Wavefront Sensor (PWFS) control, conducted within the MICADO camera SCAO module ${ }^{1,2}$ development team at LESIA. Developing a PWFSbased Adaptive Optics (AO) system with more than $80 \times 80$ resolution for the E-ELT brings up unprecedented scalability challenges to state-of-the-art focal plane sensing, making of paramount importance the current effort to gather experience, data, and thorough knowledge of PWFSs.

Since its introduction as a linearized Foucault knife-edge test with user-tunable sensitivity/dynamic trade-off, ${ }^{3}$ the PWFS was demonstrated to provide a valuable sensitivity increase over equivalent SH sensors. ${ }^{4,5}$ Many currently developed high order, high sky coverage AO systems rely on the use of one or several PWFS, including instrumental upgrades of $10 \mathrm{~m}$ class telescopes and first light instruments of all the projected 30-40 meter class telescopes.

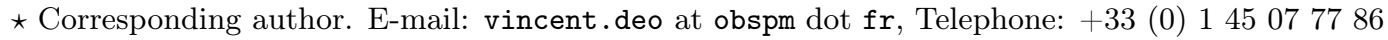


However, theoretical and experimental developments on the PWFS have brought up much debate and are an active research topic. Knowledge on modulation, ${ }^{6}$ photon efficiency, ${ }^{7}$ theoretical models, ${ }^{8-10}$ optimal modal control, ${ }^{11,12}$ or phase reconstruction algorithms does not yet converge into a unique, well established set of operation guidelines.

This paper presents a possible improvement to PWFS control, named Expanded Space Control (ESC), which through expanding the dimension of the sensor data space provides an operating mode that is robust to independent translations of the four sensor quadrants, and so to several PWFS defects. This work presents a Fourier domain analytical approach to the control completeness problem, followed by end-to-end simulations on $\mathrm{COMPASS}^{13,14}$ and bench experiment cases demonstrating the performance of ESC.

\section{EXPANDED SPACE CONTROL OF THE PWFS}

Imaging the telescope pupil (or altitude layer meta-pupil) through a PWFS generates 4 pupil-like images (quadrants) in the sensor plane, typically as on Fig. 1 . These $A, B, C$ and $D$ quadrants can generally be assumed optically independent, whether by using a prism with sufficient light deviation or with a modulation angle large enough for a favorable in-quadrant to edge-diffracted light ratio. Pyramid signals are preprocessed from sensor image data. Pupil quadrants $A, B, C$ and $D$ are extracted by selecting illuminated quadrant areas:

$$
\left[\begin{array}{l}
A(x, y) \\
B(x, y) \\
C(x, y) \\
D(x, y)
\end{array}\right]=\frac{1}{\iint I(x, y) \mathrm{d} x \mathrm{~d} y}\left[\begin{array}{l}
I\left(x-x_{A}, y-y_{A}\right) \\
I\left(x-x_{B}, y-y_{B}\right) \\
I\left(x-x_{C}, y-y_{C}\right) \\
I\left(x-x_{D}, y-y_{D}\right)
\end{array}\right]
$$

and cropping to the quadrant illuminated pixels, where $\left(x_{i}, y_{i}\right)_{i \in A, B, C, D}$ is the quadrant exact position on the sensor and $I(x, y)$ is the intensity map in the sensor plane. We use the normalization of signals with subaperture average flux, as introduced by Vérinaud. ${ }^{7}$ Quadrant origins $\left(x_{\bullet}, y_{\bullet}\right)$ are given by the deflection angle of prism facets, and the scaling factor provided by the pupil imaging lens. Then $[A, B, C, D]$ is processed into gradient-like slopes maps $S_{x}$ and $S_{y}$ :

$$
\left[\begin{array}{l}
S_{x} \\
S_{y}
\end{array}\right](x, y)=\left[\begin{array}{l}
A-B+C-D \\
A+B-C-D
\end{array}\right](x, y)=\left[\begin{array}{rrrr}
1 & -1 & 1 & -1 \\
1 & 1 & -1 & -1
\end{array}\right] \cdot\left[\begin{array}{l}
A \\
B \\
C \\
D
\end{array}\right](x, y) .
$$

The exact analytical nature of the slopes maps $S_{x}$ and $S_{y}$ for the perfect PWFS is not trivial and several interpretations coexist to this day. Originally, Ragazonni ${ }^{3}$ proposed a ray optics interpretation leading to $S_{x}, S_{y}$ being exact gradient components up to a saturation threshold. Vérinaud ${ }^{7}$ introduced a one-dimensional forward model of mixed behavior between derivator and direct phase sensing depending on spatial frequency. Several 1-D and 2-D forward PWFS models were developed during the last decade, e.g. by Shatokhina et al., ${ }^{8,15}$ and latest developments by Fauvarque et al. ${ }^{9}$ propose a linear Optical Transfer Function model for small signals of the PWFS and the slopes maps.

It is beyond the scope of this paper to discuss the validity bounds and interpretations of these models. However, we briefly summarize that (1) $S_{x}$ and $S_{y}$ are direction-sensitive linear operators in a small signal regime that is compatible with AO closed loop operation and (2) $S_{x}$ and $S_{y}$ contain sufficient information to perform a complete and unambiguous (besides piston mode) phase reconstruction. The latter point is key to this study, as the analytical focus is to ensure that measurements of a misaligned PWFS contain at least equivalent information to $S_{x}$ and $S_{y}$ of a perfect PWFS. 


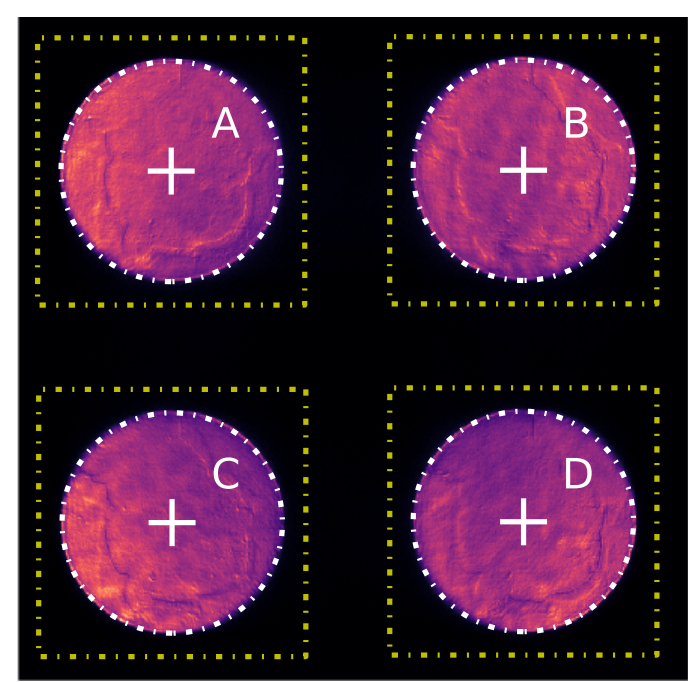

Figure 1. Typical observation in the PWFS sensor plane -here on the PYRCADO bench- illustrating requirements for quadrant registration. White: conventional control requires subpixel precision in estimating contours and centers of quadrants. Yellow: with ESC, a general selection zone containing all illuminated pixels of the quadrant is sufficient. Black pixels can be eliminated from the selection zone afterwards.

Slopes: normal(top) vs. misaligned(bottom)

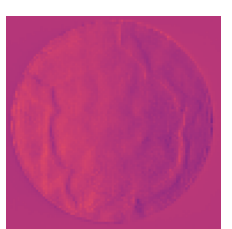

X axis slopes

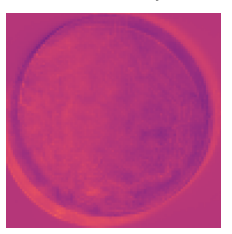

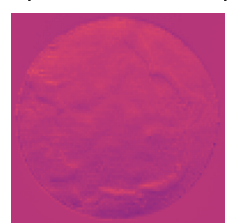

Y axis slopes

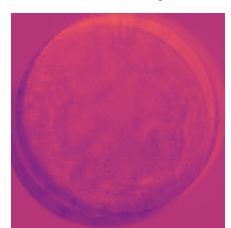

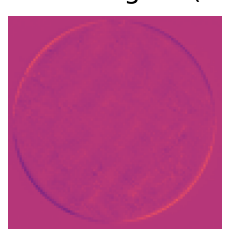

S_z cross term

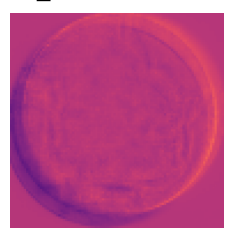

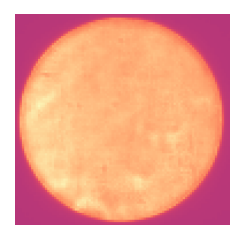
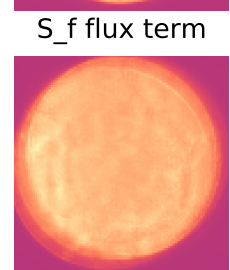

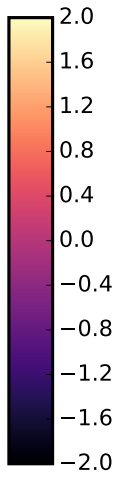

Figure 2. The 4 ESC slopes map on the PYRCADO bench (from left to right) $S_{x}^{\text {Meas. }}, S_{y}^{\text {Meas. }}, S_{z}^{\text {Meas. }}$ and $S_{f}^{\text {Meas. }}$ (Eq. 6) on a zero residual phase reference. Top row: quadrant misalignment is within $0.2 \%$ of the pupil, $S_{z}^{\text {Meas. }} \approx 0$ and $S_{f}^{\text {Meas. }}$ should be independent of the input phase. Bottom row: a strong misalignment case (approx. $5 \%$ of pupil size), illustrating how edge information is present in $S_{z}^{\text {Meas. }}$ and $S_{f}^{\text {Meas. }}$.

Expanded Space Control adds to the PWFS data space two additional slopes maps computed from quadrants:

$$
\left[\begin{array}{l}
S_{x} \\
S_{y} \\
S_{z} \\
S_{f}
\end{array}\right](x, y)=\underbrace{\left[\begin{array}{rrrr}
1 & -1 & 1 & -1 \\
1 & 1 & -1 & -1 \\
1 & -1 & -1 & 1 \\
1 & 1 & 1 & 1
\end{array}\right]}_{\mathbf{P}} \cdot\left[\begin{array}{l}
A \\
B \\
C \\
D
\end{array}\right](x, y),
$$

$S_{z}$ and $S_{f}$ respectively describing a cross-term and an out-of-reference flux term. It is to be noted that $S_{z}$ and $S_{f}$ bear no small-signal information for (1) exactly referenced quadrants, (2) a perfect 4-sided prism and (3) homogeneous and stationary pupil illumination, as demonstrated recently in Fauvarque et al. ${ }^{10}$ However, when any of conditions (1)-(3) fails, the conclusion that $S_{z}$ and $S_{f}$ are independent of the input field does not hold. An example of the four ESC slope maps in an aligned and a strongly misaligned case are shown on Fig. 2. 
When considering translations of quadrants within the sensor plane, the measurements (noted •Meas.) are related to the exact quadrants through:

$$
\left[\begin{array}{l}
A^{\text {Meas. }}(x, y) \\
B^{\text {Meas. }}(x, y) \\
C^{\text {Meas. }}(x, y) \\
D^{\text {Meas. }}(x, y)
\end{array}\right]=\left[\begin{array}{l}
A\left(x-\delta_{A}, y-\epsilon_{A}\right) \\
B\left(x-\delta_{B}, y-\epsilon_{B}\right) \\
C\left(x-\delta_{C}, y-\epsilon_{C}\right) \\
D\left(x-\delta_{D}, y-\epsilon_{D}\right)
\end{array}\right]
$$

where $\left(\delta_{i}, \epsilon_{i}\right)_{i \in A, B, C, D}$ are the sensor plane offsets between the geometrical center $\left(x_{i}, y_{i}\right)$ of the quadrant and the point estimated as such. The relationship of Eq. 4 expresses in the spatial frequency domain as:

$$
\left[\begin{array}{l}
\hat{A}^{\text {Meas. }}(u, v) \\
\hat{B}^{\text {Meas. }}(u, v) \\
\hat{C}^{\text {Meas. }}(u, v) \\
\hat{D}^{\text {Meas. }}(u, v)
\end{array}\right]=\left[\begin{array}{l}
\exp \left(2 i \pi\left(\delta_{A} u+\epsilon_{A} v\right)\right) \cdot \hat{A}(u, v) \\
\exp \left(2 i \pi\left(\delta_{B} u+\epsilon_{B} v\right)\right) \cdot \hat{B}(u, v) \\
\exp \left(2 i \pi\left(\delta_{C} u+\epsilon_{C} v\right)\right) \cdot \hat{C}(u, v) \\
\exp \left(2 i \pi\left(\delta_{D} u+\epsilon_{D} v\right)\right) \cdot \hat{D}(u, v)
\end{array}\right]
$$

where $\hat{f}(u, v)$ is the 2-D continuous Fourier transform of $f(x, y)$. Using the $\mathbf{P}$ transform (Eq. 3) from $[A, B, C, D]$ blocks to $\left[S_{x}, S_{y}, S_{z}, S_{f}\right]$ blocks, one can obtain, using $\mathbf{P}^{-1}=1 / 4 \mathbf{P}^{\mathbf{T}}$ :

$$
\left[\begin{array}{l}
\hat{S}_{x} \\
\hat{S}_{y} \\
\hat{S}_{z} \\
\hat{S}_{f}
\end{array}\right](u, v)=\underbrace{\frac{1}{4} \mathbf{P} \overline{\Delta(u, v)} \mathbf{P}^{\mathbf{T}}}_{\text {Mis }}\left[\begin{array}{l}
\hat{S}_{x}^{\text {Meas. }} \\
\hat{S}_{y}^{\text {Meas. }} \\
\hat{S}_{z}^{\text {Meas. }} \\
\hat{S}_{f}^{\text {Meas. }}
\end{array}\right](u, v)
$$

where the $\Delta$ transform is the blockwise linear phasor corresponding to quadrant translations:

$$
\Delta(u, v)=\operatorname{Diag}\left[\begin{array}{l}
\exp \left(2 i \pi\left(\delta_{A} u+\epsilon_{A} v\right)\right) \\
\exp \left(2 i \pi\left(\delta_{B} u+\epsilon_{B} v\right)\right) \\
\exp \left(2 i \pi\left(\delta_{C} u+\epsilon_{C} v\right)\right) \\
\exp \left(2 i \pi\left(\delta_{D} u+\epsilon_{D} v\right)\right)
\end{array}\right]
$$

The Mis transformation (Eq. 6) provides us with an optical transfer function (OTF) from the 4 measured slopes maps $\left[S_{x}^{\text {Meas. }}, S_{y}^{\text {Meas. }}, S_{z}^{\text {Meas. }}, S_{f}^{\text {Meas. }}\right]$ to the 2 theoretical slopes maps $\left[S_{x}, S_{y}\right]$. It is worth noting that Mis is a unitary transform with specific structure:

$$
\operatorname{Mis}(u, v)=\left[\begin{array}{llll}
p & q & r & s \\
q & p & s & r \\
r & s & p & q \\
s & r & q & p
\end{array}\right] \text { with }\left[\begin{array}{c}
s \\
r \\
q \\
p
\end{array}\right](u, v)=\frac{1}{4} \mathbf{P} \cdot\left[\begin{array}{l}
\exp \left(-2 i \pi\left(\delta_{A} u+\epsilon_{A} v\right)\right) \\
\exp \left(-2 i \pi\left(\delta_{B} u+\epsilon_{B} v\right)\right) \\
\exp \left(-2 i \pi\left(\delta_{C} u+\epsilon_{C} v\right)\right) \\
\exp \left(-2 i \pi\left(\delta_{D} u+\epsilon_{D} v\right)\right)
\end{array}\right]
$$

with coefficients additionally verifying $|p+q+r+s|(u, v)=1$ for any frequency $(u, v)$.

\section{MISALIGNMENT TRANSFORM ANALYSIS}

The Mis transformation, as a blockwise OTF, describes the information available for potential reconstruction for various control modes. We investigate how the information required for reconstruction, i.e. $\left[S_{x}, S_{y}\right]$, can be gathered from the misaligned measurements $\left[S_{x}^{\text {Meas. }}, S_{y}^{\text {Meas. }}, S_{z}^{\text {Meas. }}, S_{f}^{\text {Meas. }}\right]$.

First, Mis $(u, v)$ being unitary at all frequencies implies that unit gain at frequency $(u, v)$ in $\hat{S}_{x}$ or $\hat{S}_{y}$ is split within all 4 measured slopes maps without loss, with respective magnitudes depending of the 4 terms $p, q, r, s$ :

- $p(u, v)$ is the direct term: measurement that is true to the reference, in either $S_{x}$ or $S_{y}$. 


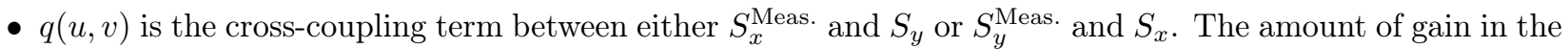
$q$ term will not impact control performance, but $S_{x}^{\text {Meas. }}$ and $S_{y}^{\text {Meas. }}$ will both contain a frequency-dependent mix of X- and Y-axis signals. Assuming $p(u, v)$ and $q(u, v)$ provide most gain at all frequencies compared to $r(u, v)$ and $s(u, v)$, no sizable performance difference is expected for conventional [ $\left.S_{x}^{\text {Meas. }}, S_{y}^{\text {Meas. }}\right]$ control.

- $r(u, v)$ and $s(u, v)$ couple $S_{x}$ and $S_{y}$ information into $S_{z}^{\text {Meas. }}$ and $S_{f}^{\text {Meas. }}$. The magnitude of these coefficients maps to the expected SNR loss for conventional control with misalignment.

We define 3 control modes for further analysis. The conventional controller (referred to as XY control) uses $\left[S_{x}^{\text {Meas. }}, S_{y}^{\text {Meas. }}\right]$ as the PWFS slope space, and two ESC modes respectively adding in the slope map $S_{z}^{\text {Meas. }}$ and both slopes maps $S_{z}^{\text {Meas. }}, S_{f}^{\text {Meas. }}$ (resp. noted XYZ and XYZF control). The test case misalignment structure and $\operatorname{Mis}(u, v)$ maps used for experiments are detailed in Sec. 4 .

\section{EXPERIMENTAL SETUP \& PROTOCOLS}

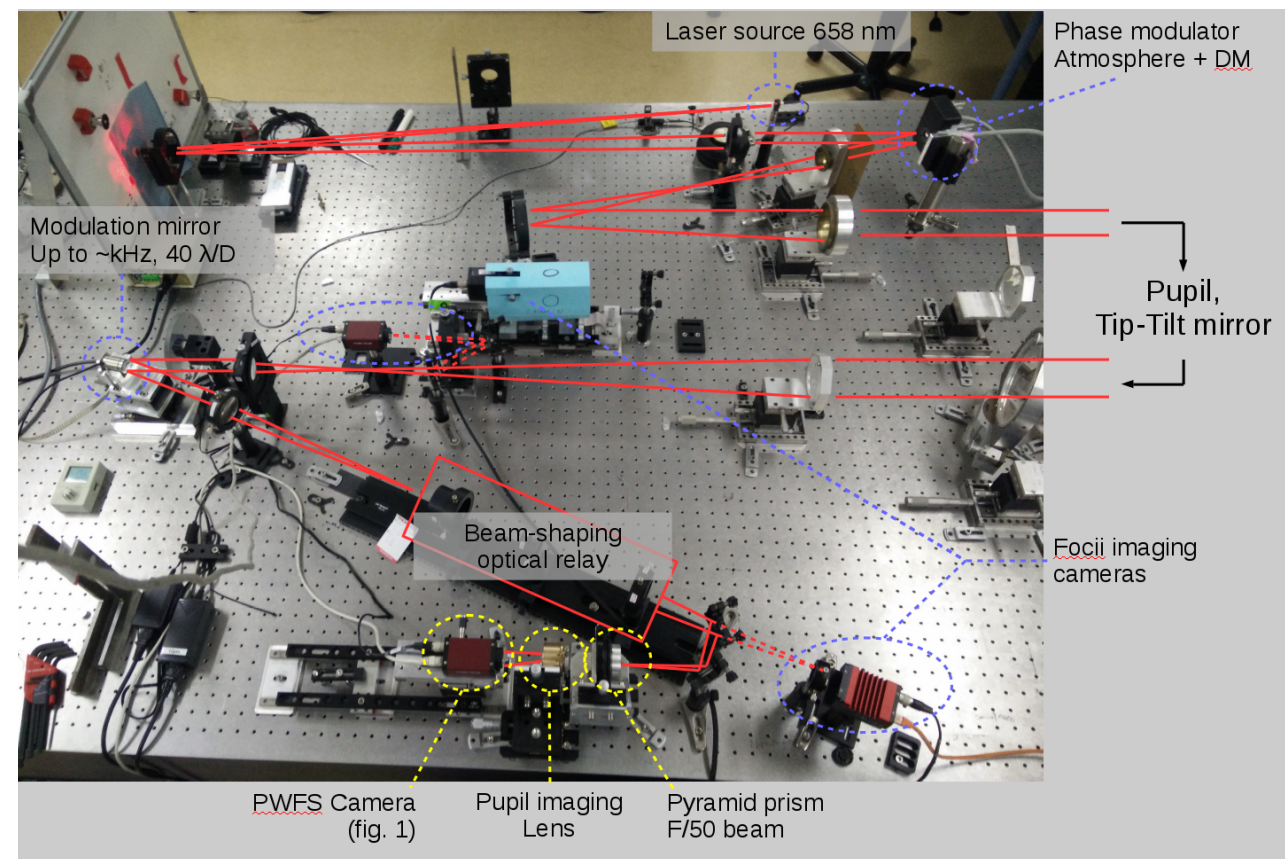

Figure 3. The PYRCADO PWFS research bench at LESIA. See text for description of elements.

The PYRamid for miCADO (PYRCADO) experiment at LESIA is a PWFS prototype developed since 2014 to study high-order AO developments and challenges, looking towards the design of high order PWFSs for ELT instrumentation.

The experiment operates with monochromatic $\lambda=658 \mathrm{~nm}$ laser light. Atmospheric distortions and high-order deformable mirrors are emulated on a Hamamatsu LCOS-SLM with a spatial resolution of $500 \mathrm{px}$ across the pupil and a depth resolution of $200 \mathrm{ADU} / \lambda$. A dedicated mirror on a physical mount compensates for tip-tilt. The WFS assembly is preceded by a fast steering tip-tilt mirror mounted on a PI oscillating piezoelectric stage which allows modulation up to a $40 \lambda / D$ angle at up to $\mathrm{kHz}$ frequency, and a beam shaping optical relay enabling $F / D$ ratios from 30 to 150 . The prism is a 4 -sided pyramid with summit half-angle $87^{\circ} 21^{\prime}$ and apex roughness $<10 \mu \mathrm{m}(0.75 \lambda / \mathrm{D}$ at F/50). Finally, quadrants are imaged with an Allied Vision Manta G-235 Gigabit ethernet camera. At aperture $\mathrm{F} / 50$ as used in experiments, quadrants are imaged with diameter $260 \mathrm{px}$, which allows for a variety of binning modes either in hardware or software.

The simulated system specifications for bench experiments and end-to-end simulations are listed in Table 1. The experimental protocol for both simulation and bench (unless otherwise specified) is the following: 
- (Bench only) Compute a phase offset map to display on the SLM to place the PWFS in Airy spot regime.

- (Bench only) Calibrate quadrant positions $\left(x_{\bullet}, y_{\bullet}\right)$ on sensor matrix, and perform a factor 6 rebin based on pixel nearest to calibrated center. Through binning, the component of $\left(\delta_{\bullet}, \epsilon_{\bullet}\right)$ due to bench alignment is maintained within $1 / 12^{\text {th }}$ of a subaperture.

- Select controller mode (XY, XYZ, XYZF) and experimental misalignments $\left(\delta_{\bullet}, \epsilon_{\bullet}\right)$

- Measure reference modal interaction matrix $I_{0}$ in Airy spot regime.

- Operate the AO loop for 0.8 sec. (400 frames at simulated $500 \mathrm{~Hz}$ ) with modal control matrix $I_{0}^{\dagger}$. The controller is ran without optical gain compensation and reaches a suboptimal stationary regime.

- Compute sensitivity loss compensation gains $G_{\text {Modal }}^{16,17}$ on closed-loop phase residual.

- Run the AO loop with gain-compensated $G_{\text {Modal }} \times I_{0}^{\dagger}$ for 0.8 sec. to reach best-effort stationary regime.

- Run the AO for 0.8 sec. more, accumulating residual wavefront and PSF data for performance results.

For repeatability of the experiment, a given misalignment pattern of the 4 quadrants was selected and then scaled to match given misalignment total tolerance. The following values of $\left(\delta_{\bullet}, \epsilon_{\bullet}\right)$ were selected, and are further referenced as the 0.5 subaperture data point in Sec. 5:

$$
\begin{aligned}
& \delta_{A}, \epsilon_{A}=-0.24,+0.46 \\
& \delta_{B}, \epsilon_{B}=+0.28,-0.49 \\
& \delta_{C}, \epsilon_{C}=-0.17,+0.38 \\
& \delta_{D}, \epsilon_{D}=+0.45,-0.47,
\end{aligned}
$$

in units of PWFS subapertures. This misalignment meets a 0.5 subaperture total tolerance, and yields the Mis transform which gain is displayed in Fig. 4. It was retained as the structure of the Mis transform makes explicit the relationships between the different slope maps. With $|q(u, v)|$ and $|r(u, v)|$ close to zero, information is split between the direct term $p(u, v)$ and the $s(u, v)$ term -coupling identically $S_{x}$ to $S_{f}^{\text {Meas. }}$ and $S_{y}$ to $S_{z}^{\text {Meas. }}{ }_{\text {. }}$. Within the two Southwest-Northeast oriented stripes where $|p(u, v)|<<1$, frequency information is exclusively contained in $S_{z}^{\text {Meas. }}$ and $S_{f}^{\text {Meas. }}$, and one can visually apprehend why using ESC is required to operate the AO system.

\section{RESULTS}

Simulations and bench runs were realized considering an $18 \mathrm{~m}$ diameter telescope with a $39 \times 39$ square-pitch deformable mirror and an approx. 35\% spatially oversampled PWFS, with a single ground layer of atmospheric turbulence of $r_{0}=12.9 \mathrm{~cm}$. The four quadrants were shifted from their true position by a scaled amount of parameters in Eq. 8, from 0 to 2.5 pixel maximum absolute value of $\left(\delta_{\bullet}, \epsilon_{\bullet}\right)$, and the AO loop was ran using XY, XYZ and XYZF modes. Detailed parameters are compiled in Table 1, and results are graphed on Figs. 5 and 7.

For both simulations and bench experiments, an analytical error budget provides us with an estimated $125 \mathrm{~nm}$ RMS fitting error, which were subtracted from the raw residual measurements. An additional budget due to photon noise, bandwidth and aliasing was computed to a total $77 \mathrm{~nm}$ RMS, shown as a dash-dotted baseline on both Fig. 5 and 7.

In simulation, all control modes provide with comparable performance for misalignments within a 0.35 subaperture upper bound. For any higher values, while XYZ and XYZF control maintain a consistent, nominal performance regardless of the misalignments, data points with XY control exhibit highly degraded performance with two possible outcomes: (1) the AO loop is unstable and the residual diverges continuously to several $\lambda$ RMS or DM saturation; or (2) the AO loop provides stable control with focal plane artifacts corresponding to blind zones in the Mis transform. Long exposure H-band PSFs for the latter cases, corresponding to starred data points in Fig. 5, are shown on Fig. 6. The speckled southwest-northeast oriented stripes on the distorted PSFs clearly illustrate the accumulation of gain cancellation stripes in the $|p(u, v)|$ Fourier plane: as the misalignment scales by a factor $f$, the periodic pattern in the $p(u, v)$ diagonal OTF term scales by a factor $1 / f$, and additional 

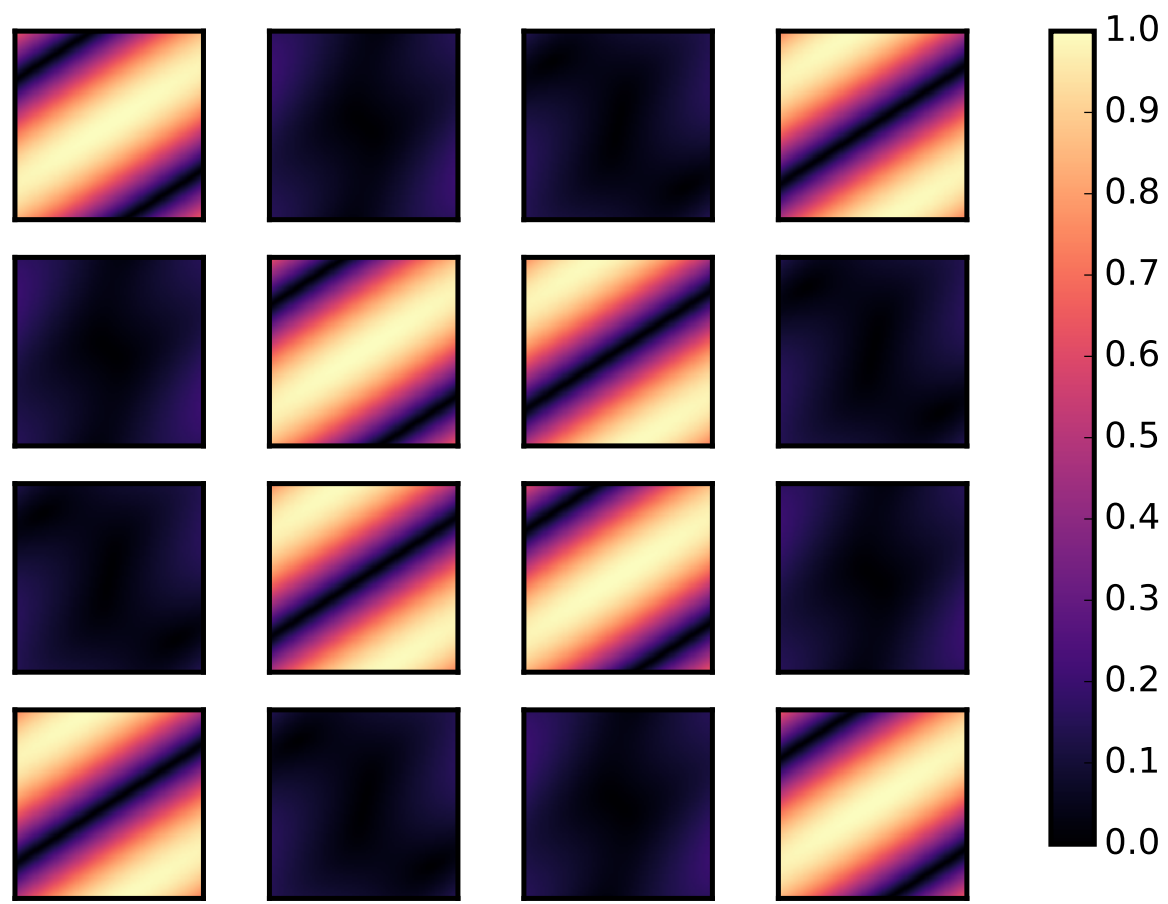

0.6

0.0

Figure 4. Frequency dependence of the magnitude of the $4 \times 4 \operatorname{Mis}(u, v)$ transform, for the misalignment test case selected for experiments (Eq. 8). Each of the 16 inserts is the magnitude of one of $p, q, r, s(u, v)$, in the order given in Eq. 7 , shown over the Nyquist domain of the PWFS: $-1 / 2 \leq u, v \leq 1 / 2$.

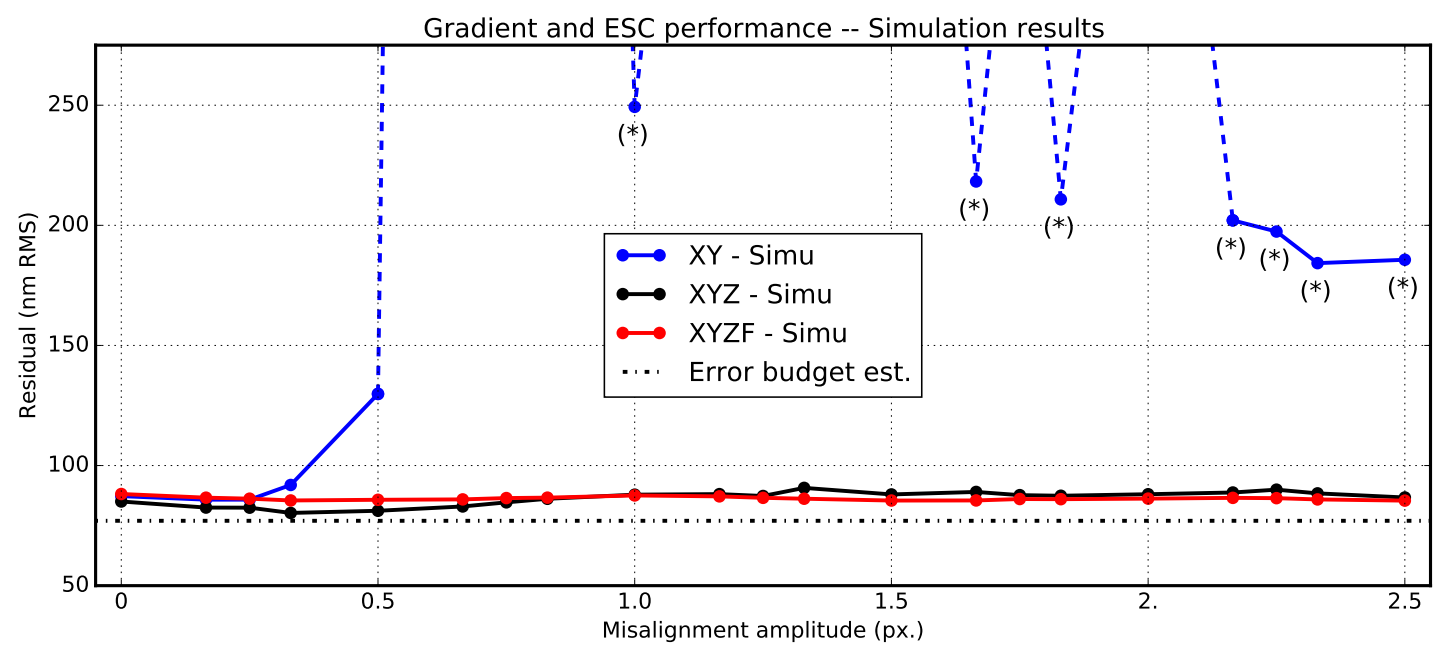

Figure 5. COMPASS end-to-end simulations of quadrant misalignment impact on AO global performance. Fitting error is already subtracted from the measured residual. Blue: conventional gradient slopes-map control. Black, Red: ESC modes. Dashed lines: AO loop diverged for intermediate points. Starred $-\left(^{*}\right)$ - points: AO loop is stable but with unsatisfactory quality; see text and Fig 6 for details. Dash-dotted line: estimated analytical error budget.

zero-gain stripes enter the Nyquist domain of the PWFS.

While XYZ and XYZF control provide equivalent performance in simulation, results of bench experiments (Fig. 7) provide us with a different conclusion. XYZF control still yields consistent performance close to $100 \mathrm{~nm}$ RMS across all misalignment values. The excess $50 \mathrm{~nm}$ RMS residual relative to simulation XYZF baseline are explained both by bench calibration and optical artifacts, mostly from the SLM. 
Table 1. Detailed simulation and bench parameters for quadrant misalignment experiments.

\begin{tabular}{|c|c|}
\hline \multicolumn{2}{|c|}{ Optical Bench \& Simulation Configuration } \\
\hline Telescope specifications & $D=18.0 \mathrm{~m}$ diameter - Circular pupil - No obstruction \\
\hline \multirow{2}{*}{ Turbulence layer } & Single Kolmogorov ground layer \\
\hline & $\mathrm{r}_{0}=12.9 \mathrm{~cm}-\mathrm{L}_{0}=25 \mathrm{~m}-\|\overrightarrow{\mathbf{v}}\|=10 \mathrm{~m} \cdot \mathrm{s}^{-1}$ \\
\hline Source & R-magnitude 13, on-axis natural guide star \\
\hline Loop rate & Simulated as $500 \mathrm{~Hz}$ \\
\hline \multirow{2}{*}{ Deformable Mirrors } & Tip-tilt mirror (bench: physical mount) \\
\hline & Square pitch piezo-stack (bench: LCOS-SLM) \\
\hline Piezo-stack mirror & 39 actuators across pupil diameter - Total 1,117 in pupil \\
\hline PWFS Subapertures & 54 across pupil $-2,290$ illuminated per quadrant image \\
\hline \multirow{2}{*}{ PWFS Wavelength } & Monochromatic at $658 \mathrm{~nm}$ \\
\hline & Photon flux of $84.2 \cdot 10^{3}$ photons per frame. \\
\hline PWFS Modulation & Circular of radius $r_{\mathrm{mod}}=8 \lambda / D$ \\
\hline \multirow{2}{*}{ Controller } & Modal integrator \\
\hline & DMs operated with 1,107 Karhunen-Loëve functions \\
\hline \multirow{2}{*}{ Controller Gain } & Integrator loop gain 0.7 \\
\hline & Modal sensitivity compensation applied (see Sec. 4) \\
\hline
\end{tabular}

H-band PSFs for nominal AO and distortion cases

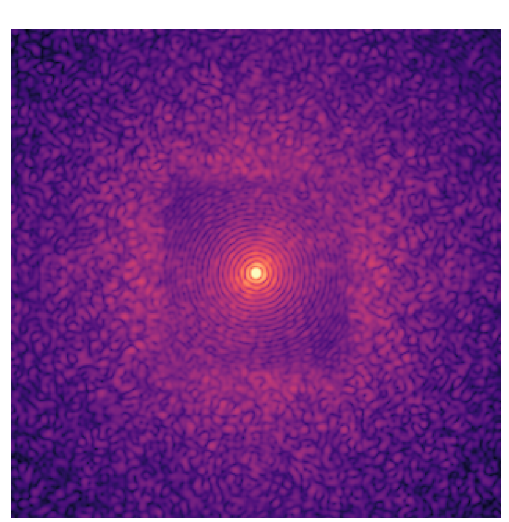

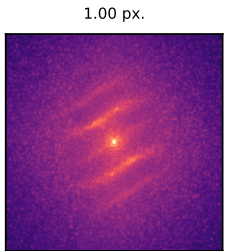

$2.17 \mathrm{px}$.

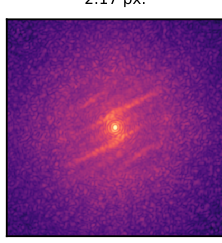

$1.67 \mathrm{px}$.

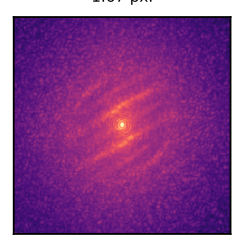

$2.33 \mathrm{px}$.

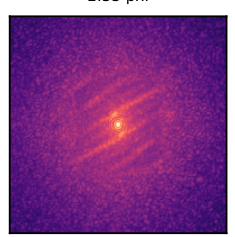

$1.83 \mathrm{px}$.

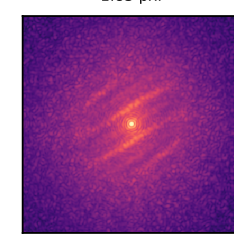

$2.50 \mathrm{px}$.

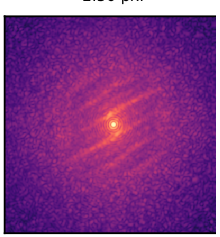

Figure 6. Log-scale representation of long exposure $(0.8$ sec.) H-band PSFs for various misalignment/controller COMPASS simulations. Left: XYZF controller with 2.5 subaperture misalignment. This PSF, with a Strehl ratio of $75 \%$, is representative of all data points in Fig. 5 in XYZ or XYZF control modes. Right inserts: PSFs for starred data in Fig. 5: for misalignments larger than 0.5 subaperture, XY control may stabilize yet the resulting PSFs exhibit strong, time-persistent stripe-like artifacts, consistent with the structure of $|p(u, v)|$. Strehl ratios for smaller inserts range from 50 to $60 \%$.

XY and XYZ control only maintain the nominal performance of approx. $100 \mathrm{~nm}$ RMS up to misalignments of respectively 0.35 and 0.7 subapertures. At higher offsets, these control modes systematically lead to AO loop instability. No cases of a stable loop yielding distorted PSFs was observed on the optical bench, vouching for the hypothesis that those cases may arise only for an extremely clean and robust AO demonstration. Stability with XYZ control should not be expected on real systems for misalignments larger than 0.7 subapertures, or even less. The observed behavior of XYZ mode is opposite between simulations and bench measurements. When XYZ allows complete misalignment robustness in simulation, the addition of the $S_{f}$ term is absolutely necessary to maintain stability on the bench. It has also been simulated that using slopes map $\left[S_{x}, S_{y}, S_{f}\right]$ provides a performance comparable to $\left[S_{x}, S_{y}, S_{z}\right]$, hinting towards a redundancy of information between $S_{z}$ and $S_{f}$ 
terms occurring only in our simulations. Whereas in simulation pupil illumination is spatially and temporally homogeneous, the bench pupil suffers from luminous flux varying both in time and space, due to: (1) pupilplane speckles, (2) non-conjugated aberrations which move in the pupil with tip-tilt, (3) interference fringes and (4) SLM phase discontinuity artifacts. The illumination variation is an additional degree of freedom that is not taken into account by the global normalization of the slopes map, and sets as indispensable to use all 4 ESC terms (or another transform of sensor data containing complete illumination information) for operating a misalignment-robust $\mathrm{AO}$ system.

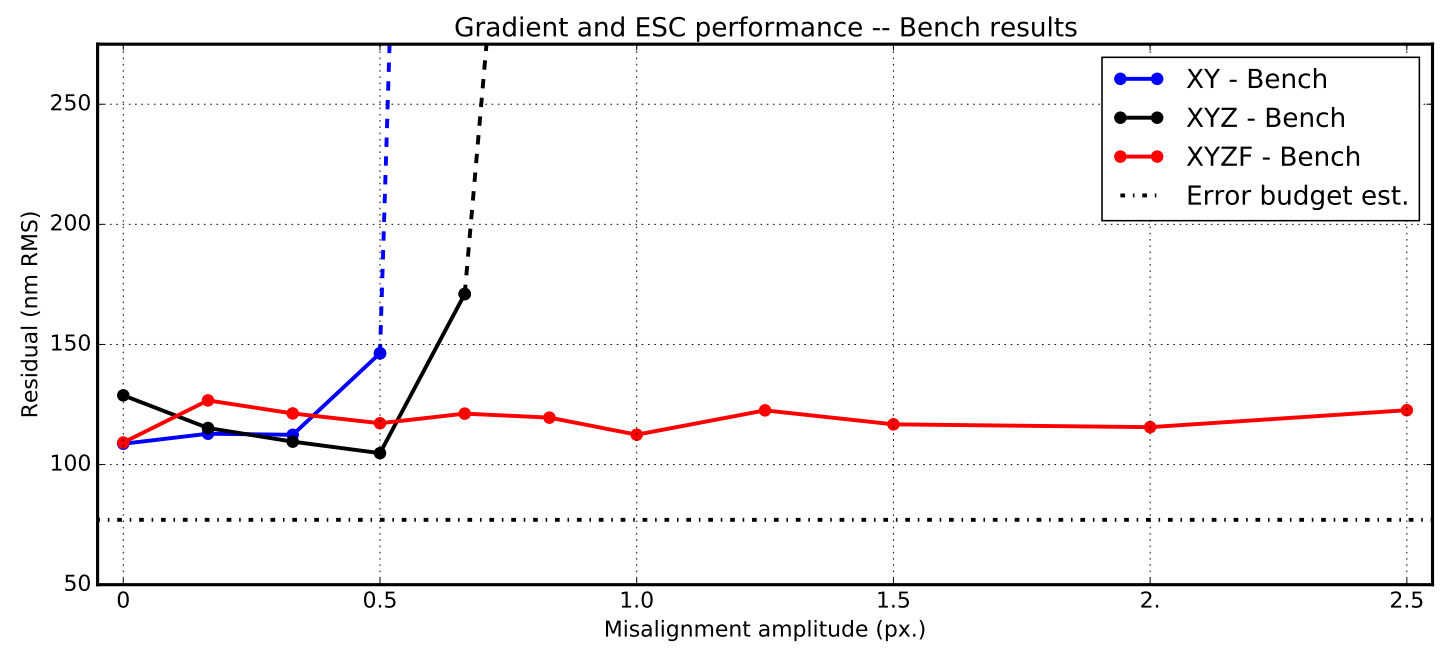

Figure 7. PYRCADO bench experiments of quadrant misalignment impact on AO global performance. Fitting error is already subtracted from the measured residual. Blue: conventional gradient slopes-map control. Black, Red: ESC modes. Dashed lines: AO loop diverged for larger misalignments.

\section{CONCLUSION \& PERSPECTIVES}

In this work, we proposed an analysis of the impact of PWFS quadrant misalignments on the sensor matrix. Such cases should naturally arise with random offsets up to 0.5 subapertures unless for an engineering effort for the PWFS to comply with tighter specifications. Larger misalignments may arise in specific situations, such as direct buffer processing of the sensor requiring memory alignment. We demonstrate, both theoretically and experimentally, that misalignments even below 0.5 subapertures may introduce significant SNR loss in the AO when conventional gradient-map control is used, and may even jeopardize AO loop stability.

We propose an alternate flavor for controlling the PWFS, by doubling the size of the measurement space. Although it is theoretically demonstrated that those extra signals do not contain information for a perfect PWFS, this is not true with quadrant misalignments, and ESC uses this additional PWFS data to gather necessary reconstructor information. Our theoretical analysis demonstrates that ESC is supposed to be insensitive to any misalignment value, and simulations and bench runs up to a 2.5 subaperture misalignment confirm this prediction. A quick analysis of different PWFS behaviors on bench and in simulation also hints towards ESC offering extra degrees of freedom in PWFS control, such as sensitivity to varying pupil illumination. In general, this study aims at bringing additional arguments to PWFS systems design trade-offs, along with prism quality and price, oversampling factor choice, RTC dimensioning and more broadly, tolerance specifications.

Finally, the authors would like to bring a quick overview of the trade-offs of using ESC found so far. First, the slope space being twice as large, instrumental RTCs should be designed to accommodate the required extra processing. It is however very accessible to mitigate this impact using parallel and/or GPU-based RTC architectures. ${ }^{18}$ The effect of switching to ESC on noise propagation through the command matrix has not yet been established and is a good candidate for further studies. So far, our experiments show no loss of performance with ESC even when alignment conditions do not require its usage, but experiments at sensitivity limit ought to be made to adjust this perspective. 


\section{REFERENCES}

[1] Davies, R., Ageorges, N., Barl, L., Bedin, L., Bender, R., Bernardi, P., Chapron, F., Clenet, Y., Deep, A., Deul, E., et al., "Micado: the e-elt adaptive optics imaging camera," Proc. SPIE 7735 (2010).

[2] Clénet, Y., Buey, T. M., Rousset, G., Cohen, M., Feautrier, P., Gendron, E., Hubert, Z., Chemla, F., Gratadour, D., Baudoz, P., et al., "Overview of the micado scao system," Proc. SPIE 9148 (2014).

[3] Ragazzoni, R., "Pupil plane wavefront sensing with an oscillating prism," J. Mod. Opt. 43(2), 289-293 (1996).

[4] Ragazzoni, R. and Farinato, J., "Sensitivity of a pyramidic wave front sensor in closed loop adaptive optics," Astron. Astrophys. 350, L23-L26 (1999).

[5] Esposito, S., Riccardi, A., Fini, L., Puglisi, A. T., Pinna, E., Xompero, M., Briguglio, R., Quirós-Pacheco, F., Stefanini, P., Guerra, J. C., Busoni, L., Tozzi, A., Pieralli, F., Agapito, G., Brusa-Zappellini, G., Demers, R., Brynnel, J., Arcidiacono, C., and Salinari, P., "First light AO (FLAO) system for LBT: final integration, acceptance test in Europe, and preliminary on-sky commissioning results," Proc. SPIE 7736 (July 2010).

[6] Ragazzoni, R., Diolaiti, E., and Vernet, E., "A pyramid wavefront sensor with no dynamic modulation," Opt. Commun. 208(1), 51-60 (2002).

[7] Vérinaud, C., "On the nature of the measurements provided by a pyramid wave-front sensor," Opt. Commun. 233(1), 27-38 (2004).

[8] Shatokhina, I., Fast Wavefront Reconstruction Algorithms for eXtreme Adaptive Optics, PhD thesis, Johannes Kepler Universität Linz (2014).

[9] Fauvarque, O., Neichel, B., Fusco, T., Sauvage, J.-F., and Girault, O., "General formalism for fourier-based wave front sensing," Optica 3, 1440-1452 (Dec 2016).

[10] Fauvarque, O., Neichel, B., Fusco, T., Sauvage, J.-F., and Giraut, O., "A general formalism for Fourier based wave front sensing: Application to the pyramid wave front sensors," (2016).

[11] Korkiakoski, V., Vérinaud, C., and Louarn, M. L., "Improving the performance of a pyramid wavefront sensor with modal sensitivity compensation," Appl. Opt. 47, 79 (2008).

[12] Gendron, E. and Léna, P., "Astronomical adaptive optics. 1: Modal control optimization," Astron. Astrophys. 291, 337-347 (1994).

[13] Gratadour, D., Puech, M., Vérinaud, C., Kestener, P., Gray, M., Petit, C., Brulé, J., Clénet, Y., Ferreira, F., Gendron, E., et al., "Compass: an efficient, scalable and versatile numerical platform for the development of elt ao systems," Proc. SPIE 9148 (2014).

[14] Carlotti, A., Vérinaud, C., Gratadour, D., Westphal, M., and Beuzit, J.-L., "Modelization of a pyramid wavefront sensor for the e-elt in the context of the compass project," Proc. SPIE 9148 (2014).

[15] Shatokhina, I., Obereder, A., Rosensteiner, M., and Ramlau, R., "Preprocessed cumulative reconstructor with domain decomposition: a fast wavefront reconstruction method for pyramid wavefront sensor," Appl. Opt. 52(12), 2640-2652 (2013).

[16] Korkiakoski, V., Vérinaud, C., Louarn, M. L., and Conan, R., "Comparison between a model-based and a conventional pyramid sensor reconstructor," Appl. Opt. 46, 6176-6184 (Aug. 2007).

[17] Korkiakoski, V., Vérinaud, C., and Le Louarn, M., "Applying sensitivity compensation for pyramid wavefront sensor in different conditions," Proc. SPIE 7015 (2008).

[18] Gratadour, D., Dipper, N., Biasi, R., Deneux, H., Bernard, J., Brule, J., Dembet, R., Doucet, N., Ferreira, F., Gendron, E., et al., "Green flash: energy efficient real-time control for ao," Proc. SPIE 9909 (2016). 